\title{
Single-stage debridement and instrumentation for pyogenic spinal infections
}

\author{
Alfred T. Ogden, M.D., And Michael G. Kaiser, M.D. \\ Department of Neurological Surgery, Columbia University, New York, New York
}

\begin{abstract}
Surgical intervention is indicated for pyogenic vertebral discitis and osteomyelitis in patients in whom medical therapy has failed, and in those with neurological compromise, mechanical instability, epidural abscess, or intractable pain. Surgical management has evolved to include single-stage operations for debridement and stabilization as well as more aggressive reconstruction strategies with respect to instrumentation. A review of the literature demonstrates excellent outcomes with single-stage operations and placement of hardware wherever it is required. Using this method, the authors have treated 16 patients without a single incidence of recurrent infection or hardware failure after almost 2 years of follow up.
\end{abstract}

KEY WORDS - spinal infection • osteomyelitis • erythrocyte sedimentation rate • C-reactive protein $\bullet$ spinal instrumentation $\bullet$ debridement

The vertebrae are the most common sites for hematogenously acquired osteomyelitis. ${ }^{38}$ Most patients with PVDO can be treated conservatively with long-term antibiotic therapy. . $^{3,4,6,7,9,20,21,35,36}$ Surgical debridement is usually reserved for patients in whom medical management of the disease has failed, those with neurological compromise, unstable mechanical deformity, an epidural abscess, or intractable pain. ${ }^{11,13,14,24,28,31}$ The decision whether to intercede surgically is often colored by the medical comorbidities endemic in this patient population. The patient's overall constitution is particularly important in these cases, given the extensive surgery often needed to stabilize the spine after debridement. Because hardware placed for fusion operations in noninfected patients has been shown to increase postoperative infection rates, many practitioners have expressed understandable concern about the placement of instrumentation in an infected patient. Historically, many have preferred to prescribe bed rest and prolonged external bracing rather than placing internal constructs. Others have advocated a staged operation with a period of antibiotic therapy bridging the debridement and instrumentation procedures. ${ }^{2,12,19,22}$ The desire to effect more rapid mobilization has led to a progressively more aggressive strategy with regard to placement of instrumentation. In recent series excellent outcomes have been demonstrated for single-stage procedures in which hardware placement is performed within and adjacent to debrided areas, and these studies have not shown significantly increased rates of infection recurrence. ${ }^{15,17,28}$

\footnotetext{
Abbreviations used in this paper: $\mathrm{CRP}=\mathrm{C}$-reactive protein; $\mathrm{ESR}=$ erythrocyte sedimentation rate; $\mathrm{PVDO}=$ pyogenic vertebral discitis and osteomyelitis.
}

\section{SPINAL INFECTIONS}

\section{Common Pathogens}

In North America, spinal infections are typically the result of methicillin-sensitive Staphylococcus aureus that spreads hematogenously in individuals with medical conditions that predispose them to infections. ${ }^{3,4,6,7,9,20,21,35,36}$ Gram-negative rods account for another large percentage and are the most prevalent infecting organisms in the intravenous drug using population. ${ }^{33}$ Brucellar vertebral osteomyelitis is common in countries where unpasteurized milk is consumed, ${ }^{3}$ and even in first world countries, tuberculous vertebral osteomyelitis comprises up to $40 \%$ of the cases in some series. ${ }^{7,9,23}$

\section{Clinical Presentation and Evaluation}

Back pain is frequently the only presenting symptom, which means that many cases of PVDO go undiagnosed for prolonged periods of time. Fever and an elevated white blood cell count are usually not present, and the diagnosis is often made when symptoms of back pain result in radiographic evaluation. When PVDO is suspected, the serum ESR and CRP level should be assayed because elevated values on these tests are more than $80 \%$ sensitive for this diagnosis. ${ }^{4,7}$ The majority of patients with spinal infections can be treated effectively with a 6- to 8-week course of antibiotic drugs and careful monitoring of neurological status, with treatment tailored to the causative organism..$^{3,4,6 \text {, }}$ $7,9,20,21,35,36$

Blood cultures are positive for bacteria in 50 to $72 \%$ of these cases..$^{3,21,23}$ When blood cultures are unrevealing, a computerized tomography-guided biopsy procedure should be the first option chosen, because this will yield 
an organism between 52 and $68 \%$ of the time, and this yield is not greatly increased in specimens obtained during open procedures. ${ }^{8,21}$

\section{Medical Management and Follow-Up Protocol}

At our institution, patients with PVDO receive a 6- to 8week course of intravenously delivered antibiotic medications, which after a time can be converted to an oral regimen in selected cases. Once an antibiotic regimen is started, patients are assessed with weekly serum ESR and CRP testing and monitored for evidence of neurological decline. The best data regarding ESR and CRP responses to antibiotic therapy are found in pediatric series of acute nonsurgically treated PVDO. In such cases, the ESR should begin to decrease in the 2nd week and to normalize within 3 to 5 weeks, whereas CRP levels should respond more rapidly, decreasing in the first couple of days and normalizing in 1 to 2 weeks. ${ }^{30,37}$

In adults, ESR trends are confounded by comorbid medical conditions and the nonspecific elevation in the rate that often occurs with age. In one study of PVDO in adults, $50 \%$ of patients who failed to demonstrate a significant decrease in the ESR after 1 month still achieved clinical cures with conservative management. ${ }^{5}$ Nevertheless, the ESR is a useful tool in the management of adult PVDO, and the authors of most studies on this disease view a 50 to $66 \%$ reduction in the ESR as compatible with eradication of infection. ${ }^{4,32}$ There are no published series of medically managed PVDO in adults in whom systematic follow-up review was performed using CRP testing.

\section{Surgical Intervention}

Most authors advocate surgical debridement in cases of failure of medical therapy, neurological compromise, or unstable mechanical deformity. ${ }^{11,13,14,24,28,31}$ The presence of an epidural abscess independent of a neurological deficit is a relatively strong indication to operate, although treatment of epidural abscesses with antibiotic drugs alone can be successful in selected cases. . $^{10,18,27,29}$ The vast majority of PVDO cases involve the anterior and/or middle spinal column. Although an epidural abscess with no significant structural deformity can be treated with simple laminectomy and evacuation, the majority of cases require an approach that allows extensive anterior debridement and reconstruction, followed by placement of a stabilization construct. This presents a conflict between the desire to place adequate instrumentation and concern over placement of a foreign body in an infected individual. These issues must be viewed in the context of the consequences of subjecting patients who are often medically ill to a second operation, prolonged periods of immobility, and weeks to months of external bracing.

Authors of retrospective series have reported outcomes obtained using strategies that reflect a continuum of treatment rationales. Investigators have advocated anterior stabilization with allograft or autograft combined with bed rest and bracing, or anterior stabilization with no instrumentation followed by a period of treatment with antibiotic drugs and a second stage for placement of a posterior construct. More recently, the trend has been to perform aggressive debridement with anterior, posterior, or circumferential stabilization at the time of the initial surgery. Some authors have also advocated removal of hardware after there is evidence of fusion to avoid the possibility of reinfection from instrumentation colonized by bacteria. Although there are no prospective data to establish the relative risks and benefits of single-stage compared with two-stage operations, the numerous retrospective series described later and in Table 1 have shown excellent outcomes with the single-stage approach.

\section{Literature Review}

In most series in which single-stage procedures for anterior spinal infections have been described, anterior debridement with placement of allograft or autograft has been used, combined with placement of a posterior stabilizing construct. This approach is based on the premise that instrumentation placed posteriorly involves a second operating field that is not (at least directly) contaminated. The first report in which this strategy was used was published by Fountain ${ }^{13}$ in 1979. Fountain presented a mixed series of patients, one of whom had destructive PVDO and who was treated with anterior corpectomy and fusion as well as posterior stabilization with Harrington rods. Although the details pertaining to the follow-up review in this individual are lacking, the fusion was apparently successful and the patient was out of bed within 14 days of the procedure.

Abramovitz, et al., ${ }^{1}$ reported a mixed series of surgically treated spinal infections in which three patients received anterior decompression and fusion with posterior placement of Harrington rods. After a follow-up duration of 1 to 9 months, two patients were ambulatory, with early evidence of bone fusion, and one had died. No other relevant follow-up data were reported.

The first series describing the consistent placement of posterior instrumentation at the time of debridement was published in 1988 by Redfern, et al. ${ }^{26}$ These authors reported on six patients with PVDO who underwent posterior placement of a Banks-Dervin rod in the same operation as a debridement procedure. Two of these patients also received anterior placement of an autologous rib strut graft. Although one patient died of pneumonia 25 days postsurgery and one died of unrelated cardiac causes 6 months later, the remaining four had "excellent" outcomes. There was one case of "troublesome" delayed wound healing but no evidence of recurrent infection.

In 1993, Graziano and Sidhu ${ }^{14}$ reported results obtained in seven patients with PVDO of the thoracic and lumbar spine who were treated with anterior corpectomy and placement of an anterior nonvascularized autogenous strut graft. Six of these patients were stabilized with a posterior fixation construct at the same surgery. The results were excellent, with no recurrence of infection, no pseudarthroses, and evidence of strut graft incorporation in all patients after a mean 25-month follow-up duration.

In 1996, Rath, et al., ${ }^{25}$ reported on a series of 43 patients with thoracic or lumbar PVDO who were treated entirely via a posterior approach. Eighteen of these patients underwent a single-stage procedure with anterior placement of autologous bone graft and posterior fixation with a transpedicular screw/rod construct. Twenty-one underwent a two-stage procedure consisting of an initial debridement, placement of gentamicin-impregnated methyl methacrylate chains in the debridement cavity, and posterior fixation, followed by an anterior fusion and removal of the 
TABLE 1

Literature review of single-stage operations for PVDO*

\begin{tabular}{|c|c|c|c|c|c|c|c|c|c|c|}
\hline \multirow[b]{2}{*}{$\begin{array}{l}\text { Authors } \\
\text { \& Year }\end{array}$} & \multirow[b]{2}{*}{$\begin{array}{l}\text { No. of } \\
\text { Patients }\end{array}$} & \multirow[b]{2}{*}{$\begin{array}{l}\text { Ant } \\
\text { Instr }\end{array}$} & \multirow[b]{2}{*}{$\begin{array}{l}\text { Pst } \\
\text { Fix }\end{array}$} & \multirow[b]{2}{*}{$\begin{array}{l}\text { Superf } \\
\text { WI }\end{array}$} & \multirow[b]{2}{*}{$\begin{array}{l}\text { Deep } \\
\text { WI }\end{array}$} & \multicolumn{4}{|c|}{ Reop } & \multirow{2}{*}{$\begin{array}{c}\text { Deaths } \\
\text { Med } \\
\text { Causes }\end{array}$} \\
\hline & & & & & & $\begin{array}{l}\text { Recurrent } \\
\text { PVDO }\end{array}$ & $\begin{array}{c}\mathrm{Hdw} / \mathrm{Graft} \\
\mathrm{Rev}\end{array}$ & $\begin{array}{l}\text { Graft } \\
\text { Extr }\end{array}$ & $\begin{array}{l}\text { Rate } \\
(\%)\end{array}$ & \\
\hline Redfern, et al., 1988 & 6 & 0 & 6 & 0 & 1 & 0 & 0 & 0 & 0 & 2 \\
\hline Graziano \& Sidhu, 1993 & 7 & 0 & 6 & NA & NA & 0 & 0 & 0 & 0 & 0 \\
\hline Rath, et al., 1996 & 18 & 0 & 18 & NA & NA & 0 & 1 & 0 & 6 & 0 \\
\hline Carragee, 1997 & 17 & 0 & 17 & 1 & 0 & 0 & 2 & 0 & 12 & 4 \\
\hline Dietze, et al., 1997 & 15 & 4 & 11 & 1 & 1 & 0 & 1 & 1 & 13 & 1 \\
\hline Safran, et al., 1998 & 10 & 0 & 10 & NA & NA & 0 & 0 & 1 & 10 & 0 \\
\hline Rezai, et al., 1999 & 26 & 14 & 12 & 1 & 2 & 1 & 2 & 2 & 19 & 0 \\
\hline Przybylski, et al., 2001 & 17 & 2 & 15 & 1 & 1 & 1 & 0 & 0 & 6 & 2 \\
\hline Hee, et al., 2002 & 21 & 6 & 10 & NA & 1 & 2 & 1 & 0 & 14 & 3 \\
\hline Liljenqvist, et al., 2003 & 20 & 20 & 20 & 4 & 0 & 0 & 2 & 0 & 10 & 3 \\
\hline
\end{tabular}

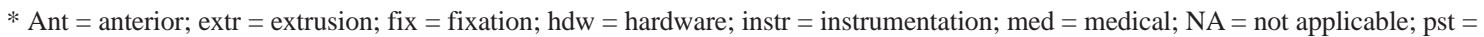
posterior; recur = recurrent; rev = revision; superf $=$ superficial; WI $=$ wound infection.

methyl methacrylate implants 2 to 3 weeks later. In the interim, patients were placed on bed rest and received intravenously administered antibiotic drugs. The authors reported no difference in outcome or morbidity rates between the two groups. There was one nonfusion in the single-stage group and there was one recurrence in the two-stage group. The recurrence occurred in one of the two patients in whom methyl methacrylate had been implanted to bridge osseous defects.

In 1997, Dietze, et al., ${ }^{11}$ reported the first series in which instrumentation was placed anteriorly, adjacent to the area of debridement. Of 20 patients with surgically treated spinal infections, 15 received instrumentation. There were four cases involving the cervical spine that were treated with anterior debridement, simultaneous arthrodesis, and placement of anterior Caspar vertebral body plates; and there were 11 cases involving the thoracic and lumbar levels that were treated with anterior debridement, arthrodesis, and posterior fixation for which either hook/rod or pedicle screw/rod constructs were used. All operations were single-stage procedures except in five of the lumbar cases, in which there was a 3- to 5-day respite between anterior decompression and arthrodesis with posterior fixation.

At the time of the report, the authors had provided follow-up care for a mean of 16 months, during which time three patients were lost to follow-up review and one patient who had multiple medical comorbidities died of septic shock. The authors reported surgical complications in four patients, including one superficial wound infection, one deep wound infection, one pseudarthrosis from a telescoped cervical interbody fibular allograft, and one dislodged lumbar interbody humeral graft that had to be replaced surgically. There were no recurrent spinal infections during the follow-up period.

In 1997, Carragee ${ }^{5}$ reported on a series of 17 consecutive patients with thoracic and lumbar PVDO who were followed for 2 years after anterior debridement and posterior fixation. There were eight "significant" postoperative complications, including two instrumentation failures and one wound dehiscence, and four patients died of medical complications. There were no recurrent infections, and ESRs returned to normal levels in 15 of the 17 patients. In two patients with "inflammatory arthritis," ESRs were halved during the follow-up period but remained in the supernormal range.

In 1998, Safran, et al., ${ }^{31}$ reported on a series of 10 patients with thoracic and lumbar PVDO who were treated with single-stage anterior corpectomy and arthrodesis. There was one repeated operation to replace an extruded anterior bone graft. After a mean follow-up duration of 30 months, all patients were ambulatory, had attained a bone fusion, and displayed no evidence of recurrent infection.

In 1999, Rezai, et al.. ${ }^{28}$ reported on a series of 57 consecutive patients with PVDO of the cervical, thoracic, or lumbar spine, 37 of whom were treated surgically. Of these, 26 underwent an anterior approach, receiving autograft or allograft, with 14 undergoing placement of anterior instrumentation alone and four undergoing anterior and posterior instrumentation. Four patients who underwent a posterior approach received fixation with hook/rod constructs. Patients were followed for a mean duration of 36 months, with none lost to follow up. Patients were treated with external bracing for 3 to 6 months postsurgery. Two patients experienced wound dehiscence that eventually healed; one patient had a superficial infection at the halo pin site. Two patients with cervical lesions who received grafts without instrumentation required a repeated operation to secure extruded bone grafts. One patient required a repeated operation to replace a screw, and another, who was noncompliant with antibiotic therapy, underwent repeated surgery for graft collapse. There was one patient who suffered a recurrent cervical infection but who nonetheless attained a solid fusion. This patient was successfully treated with abscess drainage, removal of the hardware, and another course of antibiotic medications.

In 2001, Przybylski and Sharan ${ }^{24}$ presented a series of 17 consecutive patients with PVDO who were treated surgically. Two patients had cervical disease, one thoracic, and 14 lumbar. All patients underwent single-stage anterior debridement and fixation, although the patient with cervical disease was the only one who received anterior instrumentation. Patients were followed for 12 to 44 months and wore external bracing for 2 to 3 months.

There were two deaths in the immediate postoperative period, one caused by pneumonia and multiorgan failure and the other by myocardial infarction. There was 
one wound dehiscence that healed by secondary intention without surgical debridement; there was one late superficial infection of a drain site that was treated with intravenous antibiotic medications. After surgical treatment of a thoracolumbar lesion, a paraspinal abscess and a new cervical PVDO developed 17 and 28 months, respectively, after the initial operation in one patient. His instrumentation was removed, a solid fusion was found, and results of the intraoperative cultures were negative. Another patient had a late deep wound infection that was treated with drainage, local wound care, and intravenously administered antibiotic drugs. Her hardware was removed several months later. Levels of CRP had normalized in all of the surviving patients after 3 months, except in three with "chronic" PVDO.

In 2002, Hee, et al. ${ }^{15}$ reported on a series of 21 patients treated via anterior approaches for 11 lumbar lesions, nine thoracic lesions, and one cervical lesion. Anterior fusion was performed in all cases, with five patients receiving a titanium mesh cage packed with autograft instead of a bone strut graft. Anterior stabilization instrumentation was used in two patients and posterior instrumentation and fusion were performed in 10 others by using hook/rod or pedicle screw/rod constructs. Two of these posterior fusions were delayed for 1 to 2 weeks. All patients wore rigid external braces until fusion was demonstrated on $\mathrm{x}$ ray films; patients were followed for a mean duration of 67 months.

Three patients died of septic shock in the immediate perioperative period. In two patients recurrent PVDO with progressive kyphosis developed, requiring a repeated operation; in one patient a dislodged hook was found; and in another a pseudarthrosis and wound infection developed. The authors found that, compared with patients who received posterior fixation, those who did not experienced faster procedures and less blood loss, but had higher complication rates and were twice as slow to attain fusion. Patients who received titanium cages spent less time in braces and had shorter hospital stays and lower complication rates.

In 2003, Liljenqvist, et al. ${ }^{17}$ reported on a series of 20 patients with thoracic or lumbar PVDO who all underwent single-stage operations consisting of anterior debridement and reconstruction in which an expandable titanium cage was used, along with posterior fixation in which a pedicle screw/rod construct was used. All patients were followed for a mean duration of 26 months with serial radiographs and serum CRP testing. Eight patients were mobilized with no external support, whereas 12 patients wore a brace for 3 to 6 months.

Three patients died during the follow-up period, two of sepsis in the postoperative period and one of a myocardial infarction that occurred after discharge. There was one common iliac vein injury that required intraoperative repair. There were two repeated operations for hardware malpositioning that had produced neurological symptoms. There were four superficial wound infections requiring surgical debridement but no deep wound infections or cases of recurrent PVDO. The CRP values had completely normalized in 11 of 20 at follow-up review, and ranged from 0.7 to $2.3 \mathrm{mg} / \mathrm{dl}$ in the remaining nine.

In 2003, Shad, et al., ${ }^{34}$ presented a series of five patients with a small cervical PVDO, all of whom were treated with anterior corpectomy and plate fixation. In four of the five patients the causative pathogen was $S$. aureus. When the authors removed and cultured the hardware after successful fusion and "cure," S. epidermidis grew in each case.

\section{Current Management Protocols}

The majority of spinal infections can be managed medically without surgical intervention. A diagnosis is made based on the results of radiographic studies and is confirmed by elevated serum ESRs and CRP levels. Identification of the pathogenic organism is accomplished using blood cultures or computerized tomography-guided biopsy sampling. Surgery is reserved for patients with neurological compromise, failed medical management, unstable mechanical deformity, epidural abscess, or intractable pain.

In reviewing our own series of patients who were surgically treated for PVDO over the last 3 years, we have identified 16 consecutive patients who underwent singlestage placement of both anterior and posterior instrumentation after debridement. Two of these patients died in the perioperative period, and four were lost to follow up. The remaining patients were followed with weekly ESR and CRP testing during antibiotic treatment, and they underwent ESR and CRP assessments 1 month after termination of antibiotic therapy. After 1 to 2 years of follow-up review, all ESRs and CRP values were normal or close to normal, and all patients demonstrated stable constructs on dynamic plain x-ray films. There were no hardware revisions and no recurrences.

\section{CONCLUSIONS}

A definitive recommendation regarding the optimal surgical strategy for PVDO is not possible given the retrospective nature of the literature and the inherent heterogeneity among case series. Still, it would appear that single-stage operations do not predispose patients to recurrent infection, at least not during a follow-up duration of 1 to 2 years. Although no study has been conducted to examine systematically the benefits of early mobilization in this population, it is logical to assume lower rates of complications such as pneumonia and deep venous thrombosis in patients undergoing single-stage operations.

There are no obvious advantages to rigorous avoidance of hardware placement into debridement cavities. Indeed, the sporadic cases of extrusion of anteriorly placed grafts reported in series in which only posterior hardware was placed indicates that anterior plate placement or fixation should be used if possible. Interestingly, in rare cases in which reexploration is performed for recurrent infection, a solid arthrodesis is often observed. This may result from the enhanced inflammatory response produced by an infectious process that is active during the initial phase of bone healing. ${ }^{16}$

Complete eradication of infection should be verified by postoperative normalization of ESRs and CRP levels. Trends in these values are greatly affected by concomitant medical conditions and the inflammatory response to surgery. Follow-up magnetic resonance imaging may be useful as well, but interpretation of these images is made difficult by the presence of enhancing noninfected granulation tissue and artifacts from the hardware. The benefits of hardware removal after fusion are unclear, and the one 
small series in which hardware colonization by a skin flora that was not the original pathogen was reported does not support the systematic removal of hardware after debridement and stabilization for PVDO.

\section{References}

1. Abramovitz JN, Batson RA, Yablon JS: Vertebral osteomyelitis. The surgical management of neurologic complications. Spine 11:418-420, 1986

2. Arnold PM, Baek PN, Bernardi RJ, et al: Surgical management of nontuberculous thoracic and lumbar vertebral osteomyelitis: report of 33 cases. Surg Neurol 47:551-561, 1997

3. Belzunegui J, Del Val N, Intxausti JJ, et al: Vertebral osteomyelitis in northern Spain. Report of 62 cases. Clin Exp Rheumatol 17:447-452, 1999

4. Beronius M, Bergman B, Andersson R: Vertebral osteomyelitis in Goteborg, Sweden: a retrospective study of patients during 1990-95. Scand J Infect Dis 33:527-532, 2001

5. Carragee EJ: Instrumentation of the infected and unstable spine: a review of 17 cases from the thoracic and lumbar spine with pyogenic infections. J Spinal Disord 10:317-324, 1997

6. Carrega G, Arena S, Bartolacci V, et al: [Non-tubercular vertebral osteomyelitis: diagnosis and therapy of 45 patients from a single Italian centre.] Infez Med 11:183-188, 2003 (Ita)

7. Chelsom J, Solberg CO: Vertebral osteomyelitis at a Norwegian university hospital 1987-97: clinical features, laboratory findings and outcome. Scand J Infect Dis 30:147-151, 1998

8. Colmenero JD, Jimenez-Mejias ME, Reguera JM, et al: Tuberculous vertebral osteomyelitis in the new millennium: still a diagnostic and therapeutic challenge. Eur J Clin Microbiol Infect Dis 23:477-483, 2004

9. Colmenero JD, Jimenez-Mejias ME, Sanchez-Lora FJ, et al: Pyogenic, tuberculous, and brucellar vertebral osteomyelitis: a descriptive and comparative study of 219 cases. Ann Rheum Dis 56:709-715, 1997

10. Darouiche RO, Hamill RJ, Greenberg SB, et al: Bacterial spinal epidural abscess. Review of 43 cases and literature survey. Medicine 71:369-385, 1992

11. Dietze DD Jr, Fessler RG, Jacob RP: Primary reconstruction for spinal infections. J Neurosurg 86:981-989, 1997

12. Emery SE, Chan DP, Woodward HR: Treatment of hematogenous pyogenic vertebral osteomyelitis with anterior debridement and primary bone grafting. Spine 14:284-291, 1989

13. Fountain SS: A single-stage combined surgical approach for vertebral resections. J Bone Joint Surg Am 61:1011-1017, 1979

14. Graziano GP, Sidhu KS: Salvage reconstruction in acute and late sequelae from pyogenic thoracolumbar infection. J Spinal Disord 6: 199-207, 1993

15. Hee HT, Majd ME, Holt RT, et al: Better treatment of vertebral osteomyelitis using posterior stabilization and titanium mesh cages. J Spinal Disord Tech 15:149-156, 2002

16. Kon T, Cho TJ, Aizawa T, et al: Expression of osteoprotegerin, receptor activator of NF- $\mathrm{KB}$ ligand (osteoprotegerin ligand) and related proinflammatory cytokines during fracture healing. J Bone Miner Res 16:1004-1014, 2001

17. Liljenqvist U, Lerner T, Bullmann V, et al: Titanium cages in the surgical treatment of severe vertebral osteomyelitis. Eur Spine J 12:606-612, 2003

18. Lyu RK, Chen CJ, Tang LM, et al: Spinal epidural abscess successfully treated with percutaneous, computed tomographyguided, needle aspiration and parenteral antibiotic therapy: case report and review of the literature. Neurosurgery 51:509-512, 2002

19. Matsui H, Hirano N, Sakaguchi Y: Vertebral osteomyelitis: an analysis of 38 surgically treated cases. Eur Spine J 7:50-54, 1998
20. McHenry MC, Easley KA, Locker GA: Vertebral osteomyelitis: long-term outcome for 253 patients from 7 Clevelandarea hospitals. Clin Infect Dis 34:1342-1350, 2002

21. Nolla JM, Ariza J, Gomez-Vaquero C, et al: Spontaneous pyogenic vertebral osteomyelitis in nondrug users. Semin Arthritis Rheum 31:271-278, 2002

22. Osenbach RK, Hitchon PW, Menezes AH: Diagnosis and management of pyogenic vertebral osteomyelitis in adults. Surg Neurol 33:266-275, 1990

23. Perronne C, Saba J, Behloul Z, et al: Pyogenic and tuberculous spondylodiskitis (vertebral osteomyelitis) in 80 adult patients. Clin Infect Dis 19:746-750, 1994

24. Przybylski GJ, Sharan AD: Single-stage autogenous bone grafting and internal fixation in the surgical management of pyogenic discitis and vertebral osteomyelitis. J Neurosurg Spine 94: 1-7, 2001

25. Rath SA, Neff U, Schneider O, et al: Neurosurgical management of thoracic and lumbar vertebral osteomyelitis and discitis in adults: a review of 43 consecutive surgically treated patients. Neurosurgery 38:926-933, 1996

26. Redfern RM, Miles J, Banks AJ, et al: Stabilisation of the infected spine. J Neurol Neurosurg Psychiatry 51:803-807, 1988

27. Reihsaus E, Waldbaur H, Seeling W: Spinal epidural abscess: a meta-analysis of 915 patients. Neurosurg Rev 23:175-205, 2000

28. Rezai AR, Woo HH, Errico TJ, et al: Contemporary management of spinal osteomyelitis. Neurosurgery 44:1018-1026, 1999

29. Rigamonti D, Liem L, Sampath P, et al: Spinal epidural abscess: contemporary trends in etiology, evaluation, and management. Surg Neurol 52:189-197, 1999

30. Roine I, Faingezicht I, Arguedas A, et al: Serial serum C-reactive protein to monitor recovery from acute hematogenous osteomyelitis in children. Pediatr Infect Dis J 14:40-44, 1995

31. Safran O, Rand N, Kaplan L, et al: Sequential or simultaneous, same-day anterior decompression and posterior stabilization in the management of vertebral osteomyelitis of the lumbar spine. Spine 23:1885-1890, 1998

32. Sapico FL: Microbiology and antimicrobial therapy of spinal infections. Orthop Clin North Am 27:9-13, 1996

33. Sapico FL, Montgomerie JZ: Vertebral osteomyelitis in intravenous drug abusers: report of three cases and review of the literature. Rev Infect Dis 2:196-206, 1980

34. Shad A, Shariff S, Fairbank J, et al: Internal fixation for osteomyelitis of cervical spine: the issue of persistence of culture positive infection around the implants. Acta Neurochir 145: 957-960, 2003

35. Stefanovski N, Van Voris LP: Pyogenic vertebral osteomyelitis: report of a series of 23 patients. Contemp Orthop 31:159-164, 1995

36. Torda AJ, Gottlieb T, Bradbury R: Pyogenic vertebral osteomyelitis: analysis of 20 cases and review. Clin Infect Dis 20: 320-328, 1995

37. Unkila-Kallio L, Kallio MJ, Eskola J, et al: Serum C-reactive protein, erythrocyte sedimentation rate, and white blood cell count in acute hematogenous osteomyelitis of children. Pediatrics 93:59-62, 1994

38. Waldvogel FA, Medoff G, Swartz MN: Osteomyelitis: a review of clinical features, therapeutic considerations and unusual aspects. 3. Osteomyelitis associated with vascular insufficiency. N Engl J Med 282:316-322, 1970

Manuscript received October 15, 2004

Accepted in final form November 4, 2004.

Address reprint requests to: Michael G. Kaiser, M.D., The Neurological Institute, 710 West 168th Street, 4th Floor, New York, New York 10032. 